\title{
On Experimental Thermal Analysis of Solid Materials
}

\author{
Pavel Koštial ${ }^{1}$, Ivo Špička ${ }^{1}$, Zora Jančíková ${ }^{1}$, Jan Valíček ${ }^{2,1}$, Marta Harničárová ${ }^{2,3}$, Josef Hlinka ${ }^{1}$ \\ ${ }^{1}$ Faculty of Metallurgy and Materials Engineering, VŠB - TU Ostrava, 17. listopadu 15, 70833 Ostrava - Poruba, \\ Czech Republic, pavel.kostial@vsb.cz, ivo.spicka@vsb.cz, zora.jancikova@vsb.cz, josef.hlinka@vsb.cz \\ ${ }^{2}$ Institute of Physics, Faculty of Mining and Geology, VŠB - TU Ostrava, 17. listopadu 15, 70833 Ostrava - Poruba, \\ Czech Republic, jan.valicek@vsb.cz, marta.harnicarova@vsb.cz \\ ${ }^{3}$ Nanotechnology Centre, VŠB - TU Ostrava, 17. listopadu 15/2172, 70833 Ostrava - Poruba, Czech Republic
}

\begin{abstract}
The paper is devoted to the presentation of a method for measurement of thermal conductivity $k$, specific heat capacity $c_{p}$, and thermal diffusivity applying the lumped capacitance model (LCM) as a special case of Newton's model of cooling. At the specific experimental conditions resulting from the theoretical analysis of the used model, we present a method for experimental determination of all three above mentioned thermal parameters for materials with different thermal transport properties. The input experimental data provide a cooling curve of the tested material. The evaluation of experimental data is realized by software, the fundamental features of which are presented here. The statistical analysis of experimental data was performed.
\end{abstract}

Keywords: Lumped capacity model, Newton's law of cooling, specific heat capacity, thermal diffusivity, thermal conductivity.

\section{INTRODUCTION}

$\mathrm{T}$ HERMAL and mechanical properties of solid state play an important role in the process of material optimization, mainly in the case of composites, concrete, polymers, steels, glass, and ceramics. Determination of fundamental thermal values such as thermal conductivity $k$, thermal diffusivity $\alpha$, and specific heat capacity $c_{p}$ cover a large spectrum of experimental methods based on direct or indirect measurements.

The cooling of objects is often described by a law, attributed to Newton, which states that the temperature difference of a cooling body with respect to the surroundings decreases exponentially with time. Such law is not generally correct but it is a good approximation for temperatures not too high and not large temperature intervals. In fact, the energy transfer from an object to its surroundings is not only due to conduction and convection but also to radiation, and the latter does not vary linearly with temperature difference, which leads to deviations from Newton's law. The papers [1]-[3] describe a history of the research on the cooling law. This history begins with a description and an interpretation of Newton's earlier work in 1701 and an overview of studies confirming or confuting Newton's law during the 18th century. Subsequently, the authors present the early studies on cooling due to radiant heat, the fundamental work of Dulong and Petit published in 1817 , and a brief overview of the research conducted after 1850 on the laws of thermal radiation and of natural and forced convection.

The article [4] studies the case of coffee cooling in a pot by means of a model realized as an Excel spreadsheet. The authors can estimate that some heat is used for evaporation, some heat is radiated to the surroundings and some amount of heat is accepted from the surroundings and we must not forget the transport of heat between liquid and pot, through the pot and finally between pot and the surroundings. They do not measure the individual heat flows, but rather put them into the model, and from comparison of the model prediction for the time-dependent temperature with the data they deduce the role of different mechanisms and the parameters which enter the model.

The work [5] is devoted to a thermal engine model which adheres to Newton's cooling law during the heat transfer processes. The thermal efficiency and its bounds at maximum output power are derived and discussed.

The study [6] consists of a numerical investigation of transient heat transfer in channel flow of an electrically conducting variable viscosity Boussinesq fluid in the presence of a magnetic field and thermal radiation. The temperature dependent nature of viscosity is assumed to follow an exponential model and the system exchanges heat with the ambient following Newton's law of cooling.

Specific properties of materials demand a large spectrum of measuring methods suitable for thermal parameter measurements. Some of them are based on a photoacoustic effect and they are described in [8].

Another group of experiments is based on the application of thermal impulse on the sample surface and its propagation through a sample. These impulse applications either have contact of the heat source with the sample or they are in a contactless (flash) mode. The fundamental theoretical work in this sense is [7]. The so-called flash method has a large spectrum of realizations [8]-[11].

Different contact impulse methods with different position of the heat source were described in [11]-[12]. The heat sources in this case usually have a meander shape.

Heat generation in the measured sample can also be realized by mechanical stress. The sample is pulled in a tensile test machine and the generated heat is caused by deformation of its structure. The temperature decay of the pre-pulled sample is measured by a thermovision camera. This measuring system allows measuring specific heat capacity, thermal diffusivity, and thermal conductivity as well as the mechanical parameters usually obtained from a tensile test [13]. The evaluation of $c_{p}$ from cooling curves was studied also in [13]-[14].

In this paper, we present a method for measurement of $k$, $c_{p}$ and $\alpha$ applying the lumped capacitance model as a special 
form of Newton's model of cooling [15]. Used method offers relatively reliable results and has a very good repeatability. Obtained results are statistically treated and they are also compared with known table values or results obtained in independent measurements.

\section{THEORETICAL BACKGROUND}

Lumped capacitance model is a special case of Newton's cooling law. In this method the internal temperature changes can be neglected, thus temperature of sample $T$ is function only of time $t$. It can be mathematically described by (1)

$$
T=T(t)
$$

A heat given to a sample can be found in the form of (2)

$$
Q=c_{p} \cdot \Delta T
$$

where $c_{p}$ is the specific heat capacity of a sample and $\Delta T$ is the temperature increase during a heat transfer.

For a heat flow from a sample to an environment with temperature $T_{\infty}$ Newton's cooling law can be written in the form (3)

$$
\frac{d Q}{d t}=-h_{t} \cdot S \cdot\left(T-T_{\infty}\right)
$$

where $Q$ is the heat given to a sample, $h_{t}$ is the total heat transfer coefficient of a sample, $S$ is the total heat flow area, $T_{\infty}$ is the environment temperature.

For the lumped capacitance method solution of surface temperature $T$ can be found as (4)

$$
m \cdot c_{p} \frac{d T}{d t}=-h_{t} \cdot S \cdot\left(T-T_{\infty}\right)
$$

further (5)

$$
m=\rho \cdot V=\rho \cdot S \cdot L
$$

where $\rho$ is density of the sample, $L$ is sample thickness and $S$ is the effective cooled surface. In our case the sample is cooled on both sides and so we can write (6)

$$
S^{*}=S_{\text {top }}+S_{\text {loverr }}=2 S
$$

After integration we subsequently obtain relations (7), (8)

$$
\begin{gathered}
\int_{0}^{t} \frac{\rho S L c d T}{\left(T-T_{\infty}\right)}=-2 h_{t} \cdot S \cdot \int_{0}^{t} d t \\
T=\left[\rho S L c \ln \left(T-T_{\infty}\right)\right]_{0}^{t}=\left[-2 h_{t} S t\right]_{0}^{t}
\end{gathered}
$$

If we set (9)

$$
\tau=\frac{\rho \cdot c_{p} \cdot L}{2 \cdot h_{t}}
$$

then (10)

$$
\left[\left(T-T_{\infty}\right)\right]_{0}^{t}=\left[e^{-t / \tau}\right]_{0}^{t}
$$

and finally for master function one can write (11)

$$
T=\left[\left(T-T_{\infty}\right)\right]_{0}^{t} \cdot \exp (-t / \tau)+T_{\infty}
$$

Relation among $k, c_{p}$ and $\alpha$ has the form as in (12)

$$
k=\alpha \cdot \rho \cdot c_{p}
$$

where $k$ is sample thermal conductivity, $\alpha$ is diffusivity, $c_{p}$, is specific heat capacity and $\rho$ is the sample density.

Validity of the model is verified by the Biot number $B i$ in the form (13)

$$
B i=\frac{h_{t} \cdot L}{2 k}<<1
$$

where (14)

$$
h_{t}=h_{c}+h_{r}
$$

represents the so called combined heat transfer coefficient through convection with the coefficient of $h_{c}$ and radiation with the coefficient of $h_{r}$.

In cases when the heat difference between the cooling body and the surrounding space is small, the combined heat transfer coefficient ht must be accepted as a constant which does not depend on temperature or time. The analysis of simulation results has shown that the radiation heat transfer coefficient observed at the temperature range of $25-26{ }^{\circ} \mathrm{C}$ is practically a linear function of temperature with increasing trend. However, the value of $\mathrm{hr}$ coefficient within the monitored temperature interval does not change by more than $0.5 \%$, which is why, taking into account the approximate $5 \%$ model accuracy, it can be approximated by its arithmetic average or median, which is less sensitive to deviation and extreme values.

The comparison shows that even at room temperatures and relatively small temperature differences between the cooling body and the surroundings, the value of radiation heat flow density is considerably high. Radiation heat flow in the given case has an average of more than $23.88 \%$ share on the total heat discharge from the surface of the body. That is why the description of the cooling process must usually take into account both convective and radiation heat transfer mechanism, and it must take into account the combined nature of the thermal interactions of the body with the surroundings, which more accurately corresponds to the macroscopic description of the heat exchange processes than during the application of a simple exponential model of the first order which neglects radiation. With increasing temperature differences between the cooling body and its surroundings, the possibility of approximation of the radiation heat transfer coefficient using a constant value is definitively lost. At higher temperatures and temperature differences, the temperature dependence of the convective heat transfer coefficient begins to show. 
Higher temperatures can also show thermal dependence of other physical parameters concentrated in the relaxation time, thus losing the character of a constant value.

\section{EXPERIMENTAL PROCEDURE}

The dimensions of the measured samples must be about $(10 \times 10 \times 2) \mathrm{mm}^{3}$ and must be finely ground. Matt black spray-paint is applied on all sides of the samples in order to ensure they have the same emissivity (approximately $98 \%$ ).

The equipment consists of a thermally insulated chamber [16] (Fig.1.).

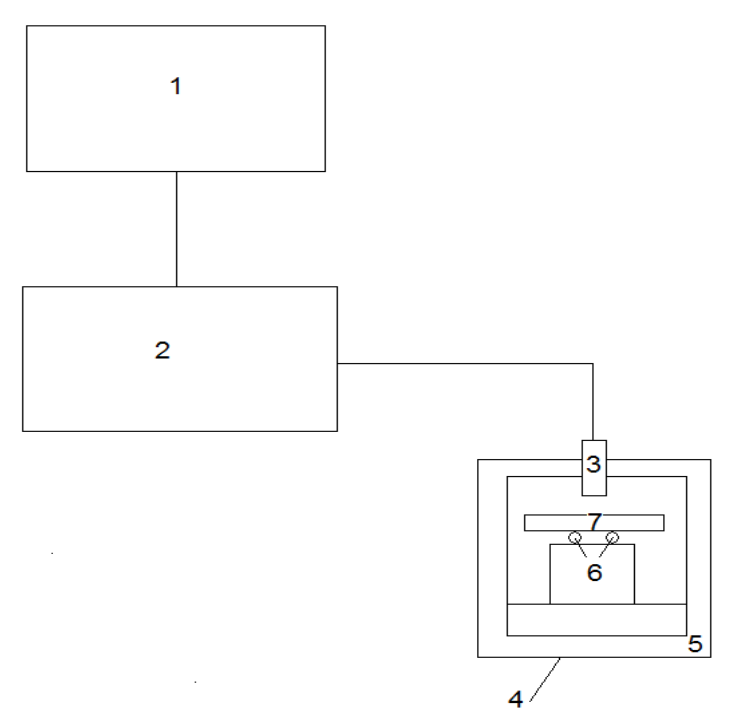

Fig.1. Schema of the apparatus: 1 - PC, 2 - electronic equipment for IR sensor and automatic data acquisition, 3 - IR sensor, 4 - adiabatic chamber, 5 - thermal isolation, 6 - feeble conductive sample support, 7 - sample [16].

The cover of the chamber has an opening for a pyroelectric sensor Raytek THERMALERT MID 02. The fundamental characteristic of the sensors is as follows. The temperature ranges from -40 to $600{ }^{\circ} \mathrm{C}$, spectral response from 8 to $14 \mu \mathrm{m}$, response time $150 \mathrm{~ms}$ (95\% response), accuracy $\pm 1 \%$ or $\pm 1{ }^{\circ} \mathrm{C}\left( \pm 2{ }^{\circ} \mathrm{F}\right)$ whichever is higher.

The sample is heated above room temperature in a thermostat $\left(40{ }^{\circ} \mathrm{C}\right)$. After removal from the thermostat, the sample is quickly placed into the measuring chamber which is then closed. It is clear that at the beginning of the sample cooling the transient process takes place. In the process of the sample cooling the relaxation time is not constant in the whole range. To judge this process we have to choose proper interval where it is approximately constant. From equation 10 we obtain (15)

$$
\tau=-t / \ln \varphi
$$

where (16)

$$
\varphi=\left[\left(T-T_{\infty}\right)-\left(T(0 s)-T_{\infty}\right)\right.
$$

Relation 15 allows setting proper, nearly constant interval from its time dependence.

Every sample has been measured ten times; average values and 99 percent confidence intervals $\left(\mathrm{P}_{99}\right)$ were calculated.

The input data are represented by the cooling curves, which are obtained by contactless temperature measurement using an infrared sensor. The set of data is automatically esampled. This data can be used to select a suitable compact curve area. This data selection also allows minimizing the radiation heat transfer coefficient $h_{r}$ (see the text above). This procedure together with adiabatic chamber construction satisfies the validity of equation 13 . The data are provided in the form of a text file with the specified parameters.

The heart of the device is software that provides data processing. The time constant is the basis for determining other heat-transport parameters of the material. It is a reason, why the program uses in the first step search for $\tau$. Equation 11, which represents the master equation of the problem, is linearized by logarithmic function and we obtain (17)

$$
\ln \left(T-T_{\infty}\right)=\ln \left(T_{0}-T_{\infty}\right)-t / \tau
$$

which is fitted by using the method of the smallest squares and the term indicates the slope of line. The initial temperature $\mathrm{T}_{0}$ is known from the measurement.

When the relaxation time $\tau$ has been determined this value is used to determine $c_{p}$ and $h_{t}$.

In the second step we begin looking for further unknown physical value $c_{p}$. Because (9) expressing the relation of $c_{p}$ versus $h_{t}$ has an infinite number of solutions, it means that only fitting procedures connected with graphical solution (see later) can be used to determine the required thermal and physical quantities. The basic prerequisite is the knowledge or at least an estimate of the total heat transfer coefficient $h_{t}$ from the material to the surroundings.

Interval $h_{t}$ has been determined from measurements of large scale of materials (high and low conductive) with the same dimensions. From these data the interval $h_{t}=(15-20)$ [W m ${ }^{-2} \mathrm{~K}^{-1}$ ] was established.

For further unknown parameter we set a qualified estimate according to the type of material - metals, plastics, laminates, etc. (Table 1.).

The size of the sample and its weight is also used to determine the density of the sample material. The algorithm in the initial step is based on the intersection of the given and possible intervals of both quantities. Once the common intervals of meaningful values have been determined, the algorithm tries to find a single value using the interval division method by gradually decreasing the interval of both required values. After this procedure $c_{p}$ is known and it is used for evaluation of thermal conductivity and thermal diffusivity. Software presents three curves: one from real measurements (cooling curve), one from upper estimate, and one from lower estimate. The curves have the following meanings. The measured values of the cooling curve of the 
sample we mark as $y_{i}$ for the points $=1 \ldots n$. The upper estimate parameters express the curve lying above the measured cooling curve, values in matching points we mark as $y_{i}^{\max }$. Lower estimate parameters express the curve lying below the measured cooling curve, its values we mark as $y_{i}^{\min }$. Squares of deviations for each measuring and estimating point are then $\left(E_{i}^{\max }\right)^{2}=\left(y_{i}-y_{i}^{\max }\right)^{2}$ for the higher estimation and $\left(E_{i}^{\text {min }}\right)^{2}=\left(y_{i}-y_{i}^{\min }\right)^{2}$ for the lower estimation, respectively.

The sum of the squared deviations is than $\sum_{i=1}^{n}\left(E_{i}^{\max }\right)^{2}$ for the higher estimation and $\sum_{i=1}^{n}\left(E_{i}^{\text {min }}\right)^{2}$ is for the lower estimation, where $n$ is the number of points of the measured cooling curve.

Table 1. Qualified estimates (intervals) of $k, c_{p}$, and $\alpha$ for some distinguished types of materials.

\begin{tabular}{|c|c|c|c|}
\hline Material & $\begin{array}{c}\text { Heat } \\
\text { capacity } \\
\text { interval } \\
\left(\mathbf{J}_{\mathbf{k g}}^{\mathbf{- 1}} \cdot \mathbf{K}^{-\mathbf{1}} \mathbf{)}\right.\end{array}$ & $\begin{array}{c}\text { Thermal } \\
\text { diffusivity } \\
\text { interval } \\
\left(\mathbf{m}^{\mathbf{2}} \cdot \mathbf{s}^{-\mathbf{1}} \mathbf{)}\right.\end{array}$ & $\begin{array}{c}\text { Thermal } \\
\text { conductivity } \\
\text { interval } \\
\left(\mathbf{W} \cdot \mathbf{m}^{-\mathbf{1}} \cdot \mathbf{K}^{\mathbf{- 1}} \mathbf{)}\right.\end{array}$ \\
\hline HDPE & $1800-2700$ & $(1.76-3.07) \cdot 10^{-7}$ & $0.46-0.52$ \\
\hline Aluminum & $866.7-908.7$ & $(0.997-1.02) \cdot 10^{-4}$ & $238.7-244.6$ \\
\hline Copper & $315-470$ & $(1-1.35) \cdot 10^{-4}$ & $380-420$ \\
\hline $\begin{array}{c}\text { Epoxide }+ \\
\text { glass fibers }\end{array}$ & $1000-1500$ & $(1-10) \cdot 10^{-7}$ & $0.1-2$ \\
\hline $\begin{array}{c}\text { Epoxide }+ \\
\text { carbon fibers }\end{array}$ & $500-2000$ & $(2-10) \cdot 10^{-7}$ & $0.1-3$ \\
\hline PC & $1000-1200$ & $(1.2-1.5) \cdot 10^{-7}$ & $0.19-0.22$ \\
\hline ABS & $1000-1200$ & $(1-1.25) \cdot 10^{-7}$ & $0.165-0.175$ \\
\hline PMMA & $1300-1500$ & $(0.9-1.1) \cdot 10^{-7}$ & $0.18-0.20$ \\
\hline Rubbers & $1200-1600$ & $(1-3) \cdot 10^{-7}$ & $0.1-0.3$ \\
\hline
\end{tabular}

For easier and more precise orientation in the process of fitting, the sum of deviation quadrates of these trajectories with the original cooling curve of the material is calculated in the software. The smallest sum $E^{2}$ indicates the best fit of both experimental and estimated curves.

Similar procedure is used for further unknown pair of thermal parameters $\alpha$ and $\mathrm{k}$, where the following equation is applied $\alpha \rho c_{p}=k, B c_{p}=k, B=\alpha \rho$ and where for both quantities qualified estimates according to the type of material at the beginning of fitting will be used (Table 1.).

Fig.2. shows the window where you can enter the qualified estimates of $h_{t}, k, c_{p}$, and $\alpha$. Fig.3. shows a window enabling you to track the difference between the experimental curve and the curves calculated from the top and bottom estimates of $h_{t}, k, c_{p}$, and $\alpha$.

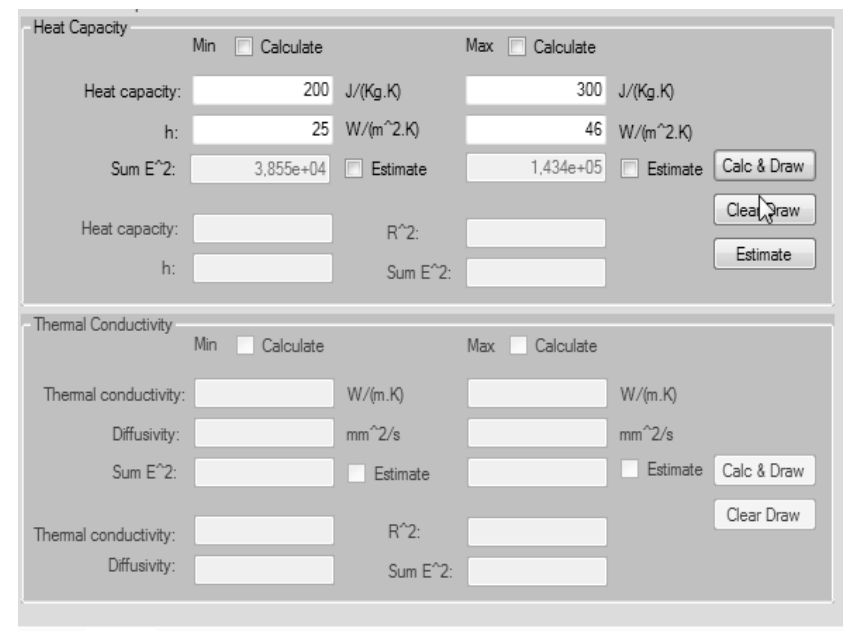

Fig.2. Print screen of the window where you can enter the qualified estimates of $h_{t}, c_{p}, k$, and $\alpha$ (bad estimate is visible also on the big values of sum $E^{2}$ ).

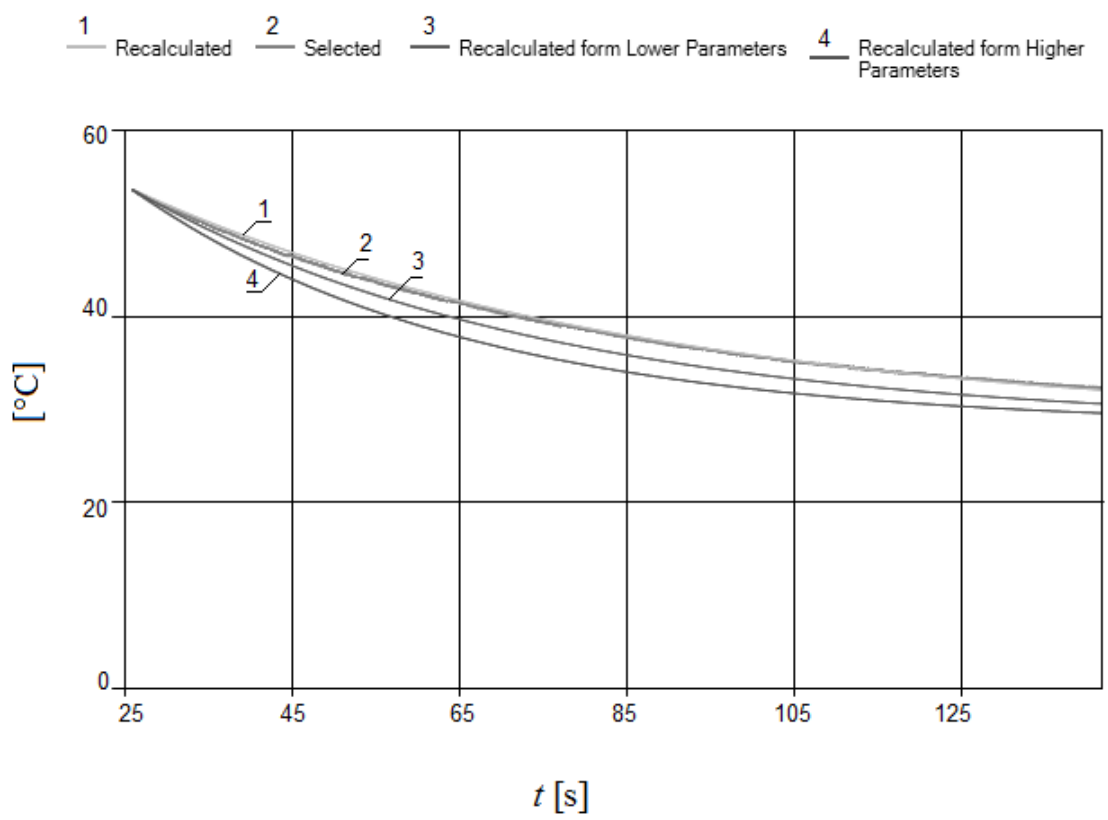

Fig.3. Print screen of a relatively good estimate shows a window enabling to track the difference between the experimental curve and the curves calculated from the top and bottom estimates of $h_{t}, k, c_{p}$, and $\alpha$ (colors of curves are not distinguished in the picture). 
In the case of materials with unknown thermal physical properties we have to start with intervals of diffusivity and thermal conductivity in the course from the lowest to higher values. The correct value we obtain by the lowest value of sum $E^{2}$

Finally, if we have determined all $k, c_{p}$, and $\alpha$ it is necessary to verify validity of the condition given by (13), which is satisfying for model validity (Biot number has to be smaller than 0.1).

In the case of very bad estimate of an interval for arbitrary physical value $k, c_{p}$, and $\alpha$, the curves obtained for the estimated interval are far from those from the measurement. Also sum $E^{2}$ is too big as it can be seen in Fig.2. The correction of the estimated interval is necessary.

On the other hand a relatively good estimate offers measured and calculated curves in the vicinity (Fig.3.).

\section{RESULTS AND DISCUSSION}

First of all we have measured the same sample of copper by the presented $L C M$ method and subsequently by Netzsch laser equipment $L F A 427$. Relative difference between both measurements of diffusivity was 8.4 percent which is a very decent result.

After this analysis, the experiment proceeds with the presentation of data obtained from selected materials with relatively high, or low value of $k, c_{p}$, and $\alpha$. Firstly, we present the measurements of thermal parameters obtained for $Z n$ and $T i$ (Tables 2. and 3.). In the next experiment we tested samples of PMMA (polymethyl metacrylate) with lower transport parameters $\alpha$ and $\mathrm{k}$ then in the previous case. Measured and table values together with statistical parameters are in Table 4.

As we can see from the presentation of results, also the conformity of the measured or tabular data is very good (relative deviations are on the level of percent), both for low-conductive material, as well as for materials with relatively higher thermal transport properties. The values of $P_{99}$ interval are calculated for measured values. The width of $P_{99}$ confidence interval is smaller than 10 percent in all samples under investigation. Biot number is smaller than 0.1 in all cases and so the used LCM is valid for a relatively wide spectrum of materials.

In [17] authors describe a seemingly similar method. Their experimental method may be summarized as follows. A thin slice of the material to be tested is held between a hot copper block and a cold copper base, which remains essentially isothermal during the measurement. After an initial transient the temperature difference between the block and the base decays exponentially, and the thermal conductivity of the sample of the material may be calculated from the exponent. Heat losses from the block to the surroundings and the influence of thermal contact resistance at the sample block interface and the sample-based interface are established by calibration. In comparison with work [17] our method offers complete set of thermal constants obtained from one cooling curve, not only $k$. Our apparatus is also able to measure materials with large scale of thermal parameters (see presented results), not only non-metals.

Table 2. Thermal parameters, tabular values and statistical parameters of zinc.

\begin{tabular}{|l|l|l|l|}
\hline \multicolumn{1}{|c|}{ Material Zn } & Measured & \multicolumn{1}{c|}{$\boldsymbol{P}_{\mathbf{9 9}}$} & Table value[19] \\
\hline$c_{p}\left[\mathrm{~J} \cdot \mathrm{kg}^{-1} \cdot \mathrm{K}^{-1}\right]$ & $410.2 \pm 5.88$ & $(391.08-429.32)$ & 385 \\
\hline$\alpha\left[\mathrm{m}^{2} \cdot \mathrm{s}^{-1}\right]$ & $(4.731 \pm 0.05) \cdot 10^{-5}$ & $(4.56-4.91) \cdot 10^{-5}$ & $4.36 .10^{-5}$ \\
\hline$k\left[\mathrm{~W} \cdot \mathrm{m}^{-1} \cdot \mathrm{K}^{-1}\right]$ & $130.8 \pm 1.70$ & $(125,26-136.34)$ & 120 \\
\hline $\mathbf{B i}$ & $\mathbf{0 . 0 4}$ & & \\
\hline
\end{tabular}

Table 3. Thermal parameters, tabular values and statistical parameters of titanium.

\begin{tabular}{|l|l|l|l|}
\hline \multicolumn{1}{|c|}{ Material Ti } & Measured & \multicolumn{1}{c|}{$\boldsymbol{P}_{\text {99 }}$} & Table value[19] \\
\hline$c_{p}\left[\mathrm{~J} \cdot \mathrm{kg}^{-1} \cdot \mathrm{K}^{-1}\right]$ & $537 \pm 6.33$ & $(516.42-557.58)$ & 520 \\
\hline$\alpha\left[\mathrm{m}^{2} \cdot \mathrm{s}^{-1}\right]$ & $(8.96 \pm 0.1) \cdot 10^{-6}$ & $(8.61-9.31) \cdot 10^{-6}$ & $8.52 .10^{-6}$ \\
\hline$k\left[\mathrm{~W} \cdot \mathrm{m}^{-1} \cdot \mathrm{K}^{-1}\right]$ & $20.8 \pm 0.25$ & $(19.99-21.61)$ & 20 \\
\hline $\mathbf{B i}$ & $\mathbf{0 . 0 3}$ & & \\
\hline
\end{tabular}

Table 4. Thermal parameters, tabular values and statistical parameters of PMMA.

\begin{tabular}{|l|l|l|l|}
\hline \multicolumn{1}{|c|}{ Material PMMA } & Measured & \multicolumn{1}{c|}{$\boldsymbol{P}_{\mathbf{9 g}}$} & $\begin{array}{l}\text { Table } \\
\text { value[19] }\end{array}$ \\
\hline$c_{p}\left[\mathrm{~J} \cdot \mathrm{kg}^{-1} \cdot \mathrm{K}^{-1}\right]$ & $1514 \pm 19.04$ & $(1452.11-1575.89)$ & 1460 \\
\hline$\alpha\left[\mathrm{m}^{2} \cdot \mathrm{s}^{-1}\right]$ & $(1.09 \pm 0.01) \cdot 10^{-7}$ & $(1.04-1.13) \cdot 10^{-7}$ & $1.07 .10^{-7}$ \\
\hline$k\left[\mathrm{~W} \cdot \mathrm{m}^{-1} \cdot \mathrm{K}^{-1}\right]$ & $0.18 \pm 0.00$ & $(0.18-0.19)$ & 0.19 \\
\hline $\mathbf{B i}$ & $\mathbf{0 . 0 4}$ & & \\
\hline
\end{tabular}




\section{CONCLUSIONS}

The presented method used to measure $k, c_{p}$ and $\alpha$ is based on Newton's model of cooling body, respectively on its modification in the form of lumped capacitance model. Software processing, based on qualified estimates of $k, c_{p}, \alpha$, and $h_{t}$, provides approximate solution of the characteristic equations, which allow relatively highly accurate determination of $k, c_{p}$, and $\alpha$. The advantage of the method is that it is contactless; it requires only small amount of tested sample, whose surface treatment is not very demanding, and finally the repeatability as well as precision of measurements is solid.

\section{ACKNOWLEDGMENT}

This work was created in the framework of the projects SP2013/29, SP2013/49, IT4Innovations Centre of Excellence project, reg. no. CZ.1.05/1.1.00/02.0070 and the project Institute of Clean Technologies for Mining and Utilization of Raw Materials for Energy Use, Reg. No. CZ.1.05/2.1.00/03.0082.

\section{REFERENCES}

[1] Besson, U. (2012). The history of the cooling law: When the search for simplicity can be an obstacle. Science \& Education, 21 (8), 1085-1110.

[2] Cheng, K.C. (2009). Some observations on the origins of Newton's law of cooling and its influences on thermofluid science. Applied Mechanics Rewiews, 62 (6), 060803.

[3] Vollmer, M. (2009). Newton's law of cooling revisited. European Journal of Physics, 30 (5), 10631084.

[4] Sedláček, J., Dolejší, J. (2010). Physical description of coffee cooling in a pot - principle of drinks cooling. In Trends in Agricultural Engineering 2010. Prague : Czech University of Life Sciences, Faculty of Engineering, 578-583.

[5] Mandelis, A. (1991). Progress in Photothermal and Photoacoustic Science and Technology. Vol. 1. SPIE.

[6] Parker, W.J., Jenkins, R.J., Butler, C.P., Abbott, G.L. (2004). Flash method of determining thermal diffusivity, heat capacity, and thermal conductivity. Journal of Applied Physics, 32 (9), 1679-1684.
[7] Gaal, P.S., Thermitus, M.A., Stroe, D.E. (2004). Thermal conductivity measurements using the flash method. Journal of Thermal Analysis and Calorimetry, 78 (1), 185-189.

[8] Akoshima, M., Baba, T. (2006). Study on a thermaldiffusivity standard for laser flash method measurements. International Journal of Thermophysics, 27 (4), 1189-1203.

[9] Ezzahri, Y., Dilhaire, S., Grauby, S., Rampnoux, J.M., Claeys, W., Zhang, Y., Shakouri, A. (2005). Study of thermomechanical properties of $\mathrm{SiSiGe}$ superlattices using femtosecond transient thermoreflectance technique. Applied Physics Letters, 87 (10), 103506.

[10] Jeng, Y.R., Chen, J.T., Cheng, C.Y. (2006). Thermal contact conductance of coated surfaces. Wear, 260 (1), 159-167.

[11] Transient Measurement Systems. (2003-2009). http://www.transientms.com.

[12] Malinarič, S., Dieška, P., Krupa, P. (2012). Modified dynamic plane source method for measuring thermophysical parameters of solids. International Journal of Thermophysics, 33 (3), 528-539.

[13] Koštial, P., Ružiak, I., Jonšta, Z., Kopal, I., Hrehuš, R., Kršková, J. (2010). Experimental method for complex thermo-mechanical material analysis. International Journal of Thermophysics, 31 (3), 630-636.

[14] Kopal, I., Kováč, K., Puchký, R. (2012). Estimation of specific heat by parametrical fitting of cooling curves. Metalurgical Journal, LXV, 7, 94-97.

[15] Lienhard, J.H. (2013). A Heat Transfer Textbook. Courier Dover Publications.

[16] Koštial, P. Ružiak, I. Jančíková, Z. (2012). Method of determining thermal conductance and specific heat capacity from drop of sample temperature and apparatus for making the same. Patent NO 303269 CS.

[17] Lisker, I.S., Solovyev, S.V., Axcell, B.P., Varlow, B.R., Donnelly, K. (2001). A transient technique for measuring the thermal conductivity of non-metals. Experimental Thermal and Fluid Science, 25 (6), 377382.

[18] Brož, J., Roskovec, V., Valouch, M.A. (1980). Physical and Mathematical Tables (in Czech). SNTL Praha. 